\title{
Revelando el signo de las cargas eléctricas usando un detector de polaridad entretenido
}

\author{
Francisco Vera ${ }^{a}$, Jaime Villanueva, Manuel Ortiz \\ Instituto de Física. Pontificia Universidad Católica de Valparaíso. Valparaíso. Chile. \\ afrancisco.vera.ucv@gmail.com
}

[Recibido: 13 diciembre 2017. Revisado: 3 febrero 2018. Aceptado: 4 marzo 2018]

\begin{abstract}
Resumen: En este trabajo discutiremos la construcción, uso y funcionamiento de un detector de polaridad electrostática que fue diseñado con el propósito de hacer interesante y entretenida una clase introductoria acerca de la existencia de cargas eléctricas y sus interacciones. El detector de polaridad usa un circuito simple y un diseño que puede imprimirse en una impresora 3D. Así el lector puede armar su propio detector luego de comprar y ensamblar de manera fácil algunos componentes electrónicos de bajo costo. El cuerpo del detector tiene la forma de un tiburón martillo cuyos ojos se encienden de acuerdo al signo de la carga del objeto que se quiere medir. Esta forma divertida de tiburón permite construir actividades entretenidas en donde los estudiantes puedan investigar las propiedades fundamentales de las interacciones entre objetos cargados eléctricamente.
\end{abstract}

Palabras clave: Electrostática; Cargas eléctricas; Electroscopio; Detector de polaridad.

\section{Unveiling the sign of electrical charges using a funny polarity detector}

\begin{abstract}
In this paper we will discuss the construction, use and operation of an electrostatic polarity detector that was designed with the purpose of making interesting and fun an introductory class about the existence of electric charges and their interactions. The polarity detector uses a simple circuit and a design that can be printed on a 3D printer. So the reader can easily build their own detector after buying and assembling some electronic components at low cost. The body of the detector has the form of a hammerhead shark whose eyes light up according to the sign of the charge of the object to be measured. This fun form of shark allows to build entertaining activities where students can investigate the fundamental properties of interactions between electrically charged objects.
\end{abstract}

Keywords: Electrostatic; Electric charges; Electroscope; Polarity detector.

Para citar este artículo: Vera F., Villanueva J., Ortiz M. (2018) Revelando el signo de las cargas eléctricas usando un detector de polaridad entretenido. Revista Eureka sobre Enseñanza y Divulgación de las Ciencias 15 (2), 2401. doi: 10.25267/Rev_Eureka_ensen_divulg_cienc.2018.v15.i2.2401.

\section{Introducción}

¿Puede la tecnología producir un cambio profundo en las clases de ciencia? Aunque la respuesta obvia es sí y los autores de este trabajo somos entusiastas de nuevos usos de la tecnología en la sala de clases, siempre hay que tener presente que el uso de la tecnología podría ir en contra de los objetivos de aprendizaje que se pretenden alcanzar. El uso de tecnología dentro del aula podría, por ejemplo, distraer a los alumnos del foco de la clase, quitar minutos valiosos de una clase al tener que esperar que los equipos funcionen de la manera esperada, complicar a los alumnos al tener que aprender particularidades de la tecnología que no son de relevancia para las materias que se pretenden enseñar, etc. Es así necesario pensar por adelantado en los posibles efectos no deseados al intentar incorporar tecnologías en el aula, pero también hay que considerar que el uso de tecnología puede ser un elemento motivador que ayude a los alumnos a sentirse parte de una clase moderna de ciencia.

En nuestra universidad el uso conjunto de nuevas tecnologías y metodologías modernas de enseñanza ha sido un elemento clave para que los cursos de laboratorio de física básica se desarrollen usando guías de carácter indagatorio y experimentos de bajo costo. El uso de vídeo

\author{
Revista Eureka sobre Enseñanza y Divulgación de las Ciencias \\ Universidad de Cádiæ. APAC-Eureka. ISSN: 1697-011X \\ bttp://dx.doi.org/10.25267/Rev_Eureka_ensen_divulg_cienc.2018.v15.i2.2401 \\ http:/ / reuredc.uca.es
}


para la medición de las coordenadas de posición de objetos en movimiento (Laws 2009, Vera 2009) y el empleo de la interfaz Arduino para la adquisición de señales desde sensores (Vera 2014) han sido elementos tecnológicos esenciales para producir este cambio. Estas tecnologías se han incorporado de manera que permitan a los alumnos usarlas en un proceso de medición simple, en donde el uso de computadores dentro de los laboratorios se ha pensado para no interferir con el desarrollo de la clase.

En contraste al uso de tecnologías que buscan pasar desapercibidas, es posible incluir elementos tecnológicos que tomen un rol protagónico dentro del aula y que buscan promover la participación de los alumnos en la clase. En este trabajo discutiremos el uso, construcción y funcionamiento de un detector de polaridad electrostática en forma de tiburón martillo que está pensado para motivar la participación de los alumnos en actividades que permiten entender conceptos fundamentales de electrostática de manera entretenida (Lozano 2013).

\section{Construcción y funcionamiento del circuito detector}

La figura 1 muestra el circuito básico de un detector de campo eléctrico publicado en 1987 (Beaty 1987) que sólo requiere de un transistor FET, un LED, una batería de $9 \mathrm{~V}$ y una resistencia de $1 \mathrm{M} \Omega$ que sirve de antena, elementos que se pueden obtener sin mucha dificultad. En un principio este detector fue propuesto para medir campos eléctricos de manera cualitativa incluso a distancias de varios metros. De acuerdo al tipo de transistor utilizado, el detector permite identificar el signo de la carga de un objeto cargado que se ubique a unos pocos centímetros de la antena del detector.
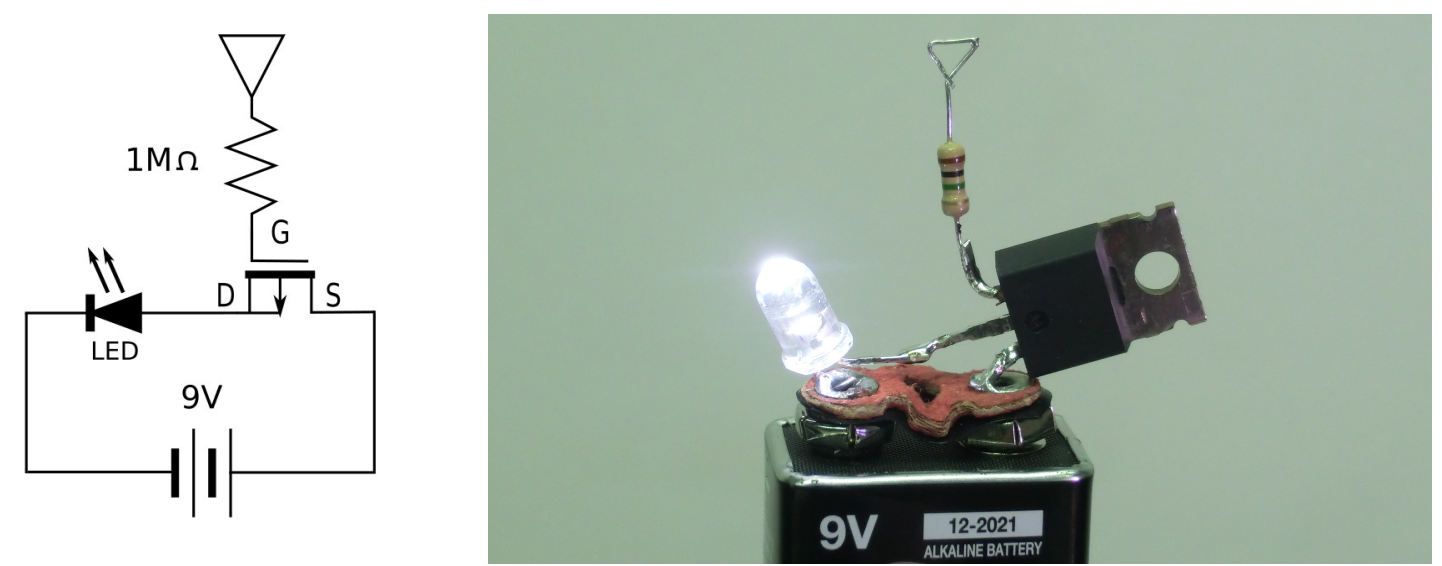

Figura 1. (Izquierda) Circuito básico del detector de campo eléctrico que consiste en un FET, una batería de $9 \mathrm{~V}$, un LED y una resistencia de $1 \mathrm{M} \Omega$ que sirve de antena. (Derecha) Los componentes instalados en un conector de batería de $9 \mathrm{~V}$.

Los transistores son la evolución de las antiguas válvulas de vacío, en donde el cambiar el voltaje de una grilla metálica ubicada entre el cátodo y el ánodo permitía modular el valor de la corriente de electrones que viajan desde el cátodo al ánodo. Usando las propiedades de los semiconductores fue posible construir transistores, dispositivos de estado sólido que permiten variar la corriente eléctrica que viaja entre el emisor y el colector al modular el voltaje de la base o compuerta. Las válvulas de vacío y los transistores se comportan como llaves de paso que permiten controlar grandes corrientes eléctricas mediante una pequeña señal de voltaje aplicada a la grilla o a la base. En particular, los transistores de efecto de campo o FET están diseñados de manera que la sola presencia de un campo en la base permite modular la corriente entre el emisor y el colector sin la necesidad de una corriente en la base. 
El circuito básico del detector de campo eléctrico funciona gracias a que los FET son muy sensibles al valor del campo en la base y es ahí en donde se ubica la antena en el circuito mostrado en la figura 1. La presencia de un campo eléctrico en la antena receptora permite variar la corriente que pasa por el LED en el circuito, haciendo que el LED emita luz o atenúe su emisión de acuerdo al signo de las cargas que producen el campo eléctrico. La resistencia de $1 \mathrm{M} \Omega$ ubicada antes de la antena permite proteger al FET de excesos de carga.

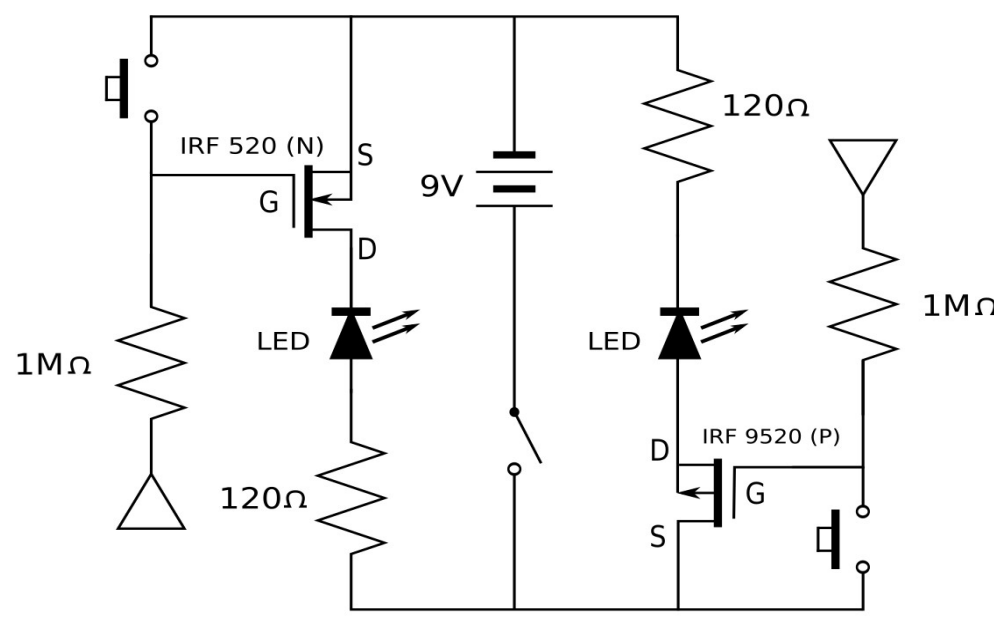

Figura 2. Circuito que permite medir la polaridad de objetos con carga positiva o negativa.
La figura 2 muestra el circuito que permite medir al mismo tiempo las polaridades positiva o negativa de objetos cargados con un alto voltaje. El circuito consiste básicamente en una versión doble del circuito publicado por W. Beaty en donde se usan MOSFET de tipo diferente para poder detectar cada uno de los signos de las cargas que producen el campo eléctrico (Dinca 2011, Isik 2015, Ganci 1994, Thompson 2014, Connolly 1991). Las resistencias de 120 $\Omega$ en nuestro circuito permiten proteger y disminuir la intensidad luminosa de cada LED haciendo que el LED respectivo se ilumine sólo bajo la presencia de un objeto cargado. Otro nuevo elemento presente en este circuito son los interruptores que permiten eliminar cargas que podrían acumularse en las antenas y que al saturar el FET respectivo mantienen el LED correspondiente permanentemente encendido, anulando el poder de detección. Es normal que se produzca una acumulación de cargas en la base del FET al detectar polaridades de objetos cargados a alto voltaje, ya que al ubicar la antena cerca de un objeto cargado es posible que el objeto deposite o saque cargas de la antena. Hay que notar que un pequeño exceso de carga en el FET hace que el circuito sea más sensible, lo que posibilita la detección de objetos cargados a mayores distancias.

\section{Un diseño divertido para el cuerpo del detector}

El diseño del detector en forma de un tiburón martillo tiene el propósito de darle un sentido lúdico al dispositivo. Esta forma fue elegida debido a que la separación entre los ojos, que se encienden de acuerdo a la polaridad de las cargas, es fácilmente visible a grandes distancias y además los tiburones son animales que pueden detectar campos eléctricos extremadamente débiles (Ampullae 2017). El diseño 3D del detector incluye un módulo separado que se incrusta en el cuerpo y que contiene los componentes electrónicos que el lector puede ensamblar de manera sencilla.

Este diseño comenzó con el Modelo-01, que consistía en el circuito mostrado en la figura 2 armado soldando los componentes, los que fueron dispuestos dentro de una caja de material plástico del tipo usado para instalaciones eléctricas sobrepuestas de uso habitacional. Luego desarrollamos el Modelo-02a mostrado en la figura 4, en donde los componentes electrónicos fueron insertados en un módulo de conexiones sin soldaduras. Con la idea de mejorar la visualización del signo de la carga y darle un sentido lúdico al detector, el modelo anterior 
evolucionó hacia el Modelo-02b al ser montado en el módulo extraíble mostrado en la figura 3 , que podía ser incrustado en un contenedor con un diseño más complejo que una simple caja rectangular. Con la finalidad de contextualizar la detección de campos eléctricos al mundo natural, se inició el diseño del Modelo-03 con una forma de tiburón martillo mostrado en la figura 3. Este modelo permite que los alumnos visualicen las conexiones que existen entre la física, la química y la biología.

El uso de componentes electrónicos soldados entre sí del Modelo-01 tiene la ventaja de hacer el circuito más robusto, pero hace difícil la búsqueda de fallas y el reemplazo de componentes. Debido a que el circuito es usado cerca de objetos a muy alto voltaje, es posible que se produzcan chispas que pueden dañar los componentes electrónicos. Por esta razón decidimos armar el circuito del Modelo-02a usando un protoboard para facilitar la búsqueda de fallas y el reemplazo de componentes. Para hacer más robusto el circuito a fallas de conexión inherentes al uso de un protoboard, diseñamos el módulo extraíble del Modelo-02b con el propósito de insertarlo dentro de un contenedor de forma arbitraria. El diseño de un detector en forma de tiburón martillo tiene claramente diversas ventajas didácticas y funcionales que, a nuestro parecer, superan desventajas como un diseño complicado y lento de imprimir en una impresora 3D.

La figura 3 muestra el cuerpo del detector imprimible en una impresora 3D en diferentes partes ensamblables, los componentes electrónicos y la batería se muestran incrustados dentro del módulo extraíble. El armado del detector es simple y no requiere de soldaduras complicadas, sólo es necesario que los terminales conectores de algunos componentes sean soldados a alambres previo al proceso de ensamblado del detector. Nuestro diseño incluye un bloque de conexiones sin soldaduras que permite reducir al mínimo las soldaduras necesarias para la obtención de un detector funcional. Luego de insertar los componentes electrónicos y de hacer pasar los cables de los LED y las antenas por unas ranuras incluidas en el cuerpo, se obtiene un dispositivo funcional.

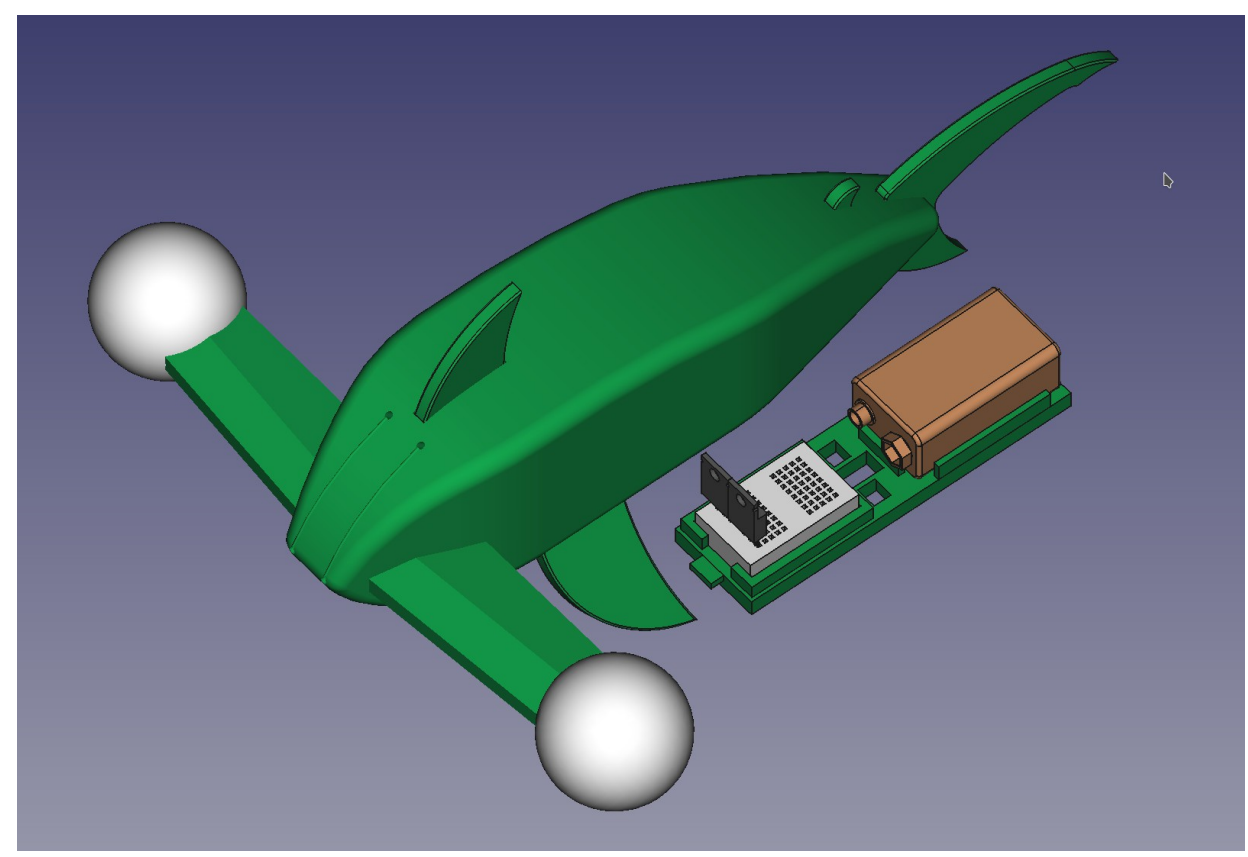

Figura 3. Modelo 3D del detector de polaridad en forma de tiburón martillo. El módulo extraíble que contiene los componentes electrónicos se muestra separado al cuerpo del detector. 
La figura 4 muestra todos los componentes electrónicos instalados en un módulo de conexiones de $22 \mathrm{~mm} \times 34 \mathrm{~mm}$ que se obtiene al cortar un protoboard para obtener dos grupos de ocho filas con cinco ranuras interconectadas. Los alambres de los LED, antenas e interruptores mostrados en la figura fueron acortados de manera que la imagen incluya todos los componentes electrónicos ensamblados y funcionando. Para armar el detector en forma de tiburón martillo, los LED se insertan dentro de pelotas de pingpong que constituyen los ojos del tiburón, luego los cables se pasan por ranuras incluidas en el diseño del cuerpo y se conectan al módulo de conexiones sin soldadura. Cada antena se pasa por su respectiva ranura y se insertan en el módulo de conexiones. Al finalizar de incrustar los componentes electrónicos mostrados en el circuito de la figura 2, se conecta la batería de $9 \mathrm{~V}$ y el detector está listo para su uso. Las únicas soldaduras necesarias para armar este circuito son las usadas para conectar alambres a los LED y a los interruptores.

Para la realización del diseño del cuerpo del detector se utilizó un software de dibujo asistido por computador de código abierto (FreeCAD 2017), disponible de forma gratuita en la web. El lector puede descargar el modelo en el formato nativo de FreeCAD y usar este programa para ajustar las dimensiones del detector en forma de tiburón acorde a sus necesidades particulares. Si el lector no desea hacer cambios en el modelo, puede descargar directamente el modelo en formato .stl y usar el software que acompaña a su impresora 3D para generar el archivo de comandos g-code que permite a la impresora imprimir capas secuenciales para generar el objeto 3D. Usando el archivo en su formato nativo, el lector puede usar el programa FreeCAD para seleccionar algún objeto del modelo y exportarlo al formato .stl para su posterior procesado por el software laminador o de slicing que genera los comandos gcode.

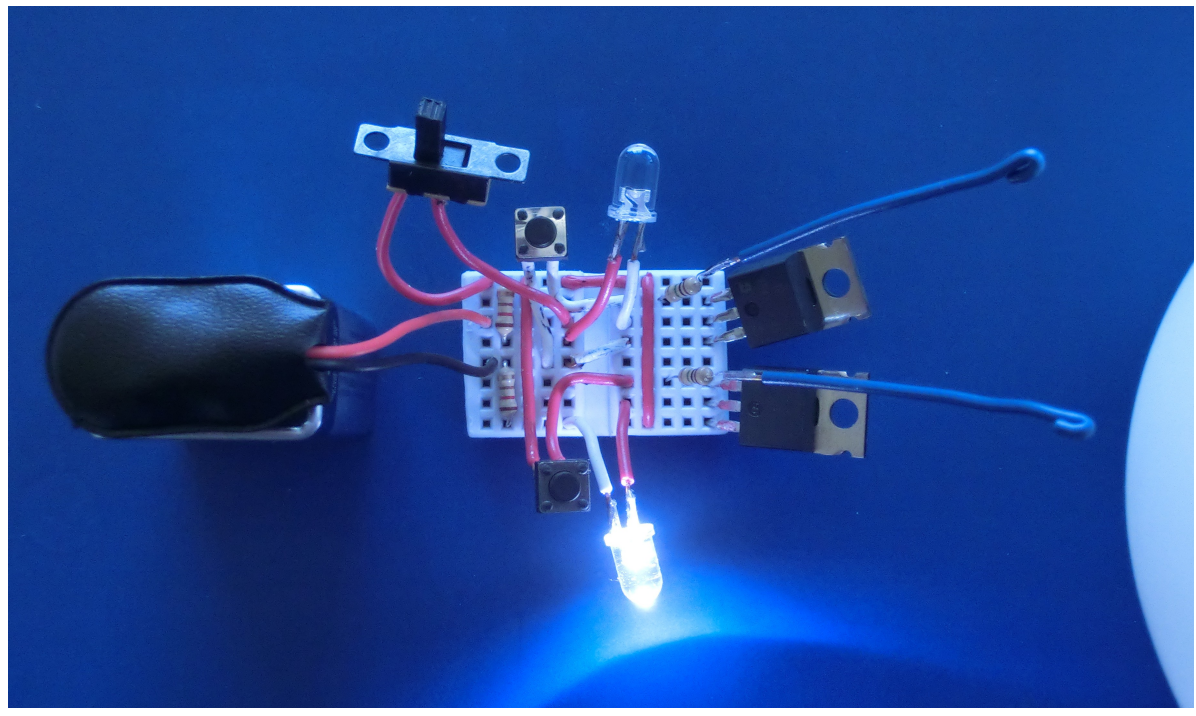

Figura 4. Componentes electrónicos instalados en un módulo de conexiones sin soldaduras (protoboard) de acuerdo al circuito mostrado en la figura 2. Este circuito se incrusta en el módulo extraíble del modelo 3D mostrado en la figura 3.

\section{Actividades para enseñar electrostática de manera entretenida usando el detector de polaridad}

En esta sección mostraremos algunos ejemplos de posibles actividades para enseñar de manera entretenida algunos conceptos fundamentales de las interacciones entre objetos cargados usando el detector de polaridad. Las actividades usan elementos simples y de bajo costo, y están dirigidas a entender el rol del signo de la carga en la atracción y repulsión de 
objetos cargados. Cabe hacer notar que todas estas actividades hacen uso de objetos cargados a un alto voltaje en donde la energía acumulada puede producir una chispa visible y en algunos casos audible pero que no es suficiente para producir daños a una persona. Se debe tener cuidado de no realizar estos experimentos cerca de artículos electrónicos y de personas que usan marcapasos o dispositivos similares. Para que los experimentos de electrostática funcionen adecuadamente se debe prestar atención a la humedad en el ambiente debido a que, cuando existe mucha humedad en el aire, los objetos que normalmente consideramos como buenos aisladores pueden conducir carga por su superficie. Es así recomendable limpiar con alcohol la suciedad de todos los objetos que queremos mantengan su propiedad de aislador además de secarlos usando aire caliente.

Levitación electrostática. Es de mucha importancia que la primera actividad de una clase de ciencias busque sorprender a los alumnos usando una actividad entretenida. Así, buscando mejorar las actitudes de los alumnos en las clases de laboratorio de física de nuestra universidad, hemos realizado al inicio de las clases de electrostática experimentos tales como: usar un globo cargado por fricción para mover una escoba sostenida por un cordel desde el techo, usar un globo cargado para hacer rodar una lata de bebida vacía sobre una mesa, levitar objetos livianos usando un generador de Van de Graaff portátil comercializado como Fun Fly Stick (Kriman 2008), etc. El hacer levitar objetos moviéndose en frente de la clase es sin dudas una actividad que entretiene y fascina a los alumnos, es además una actividad muy simple de realizar usando un globo cargado por fricción y un trozo cilíndrico de bolsa plástica muy delgada o globos muy livianos.

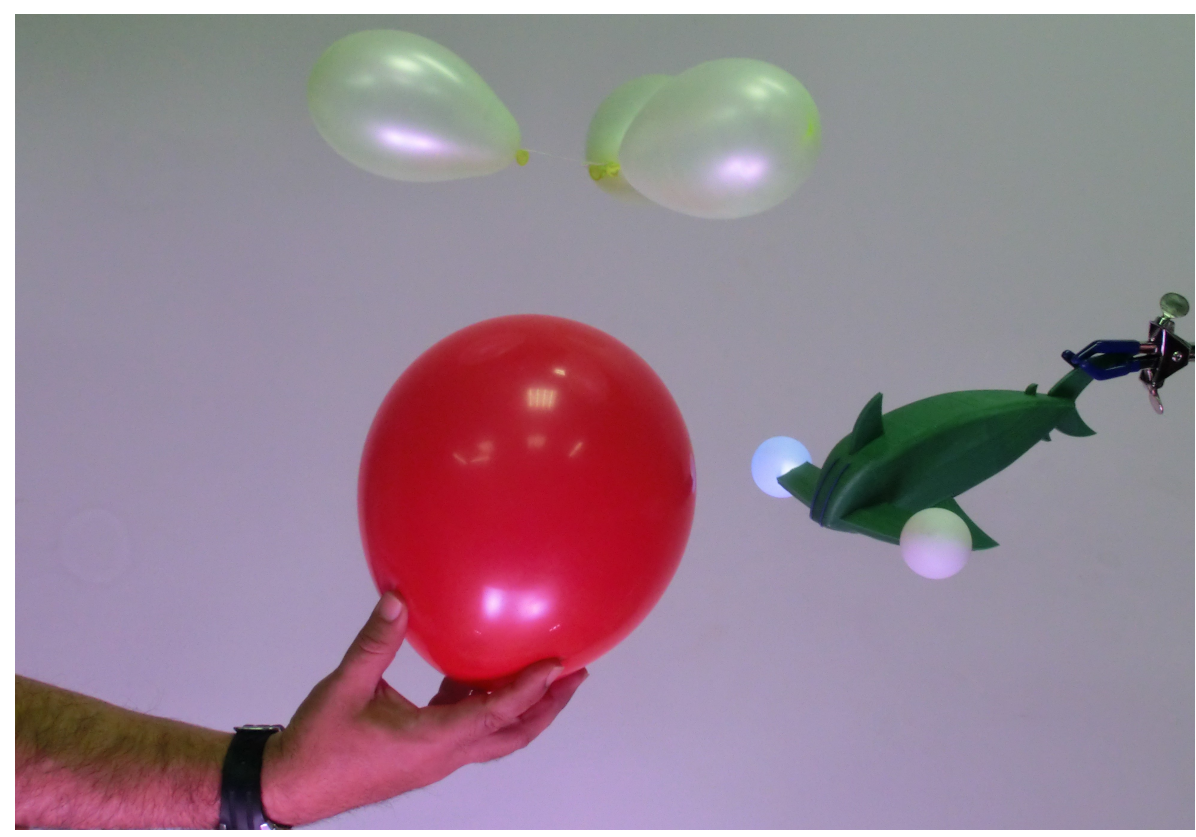

Figura 5. Levitación de tres globos cargados muy livianos unidos entre sí usando otro globo cargado con la misma polaridad.

La figura 5 muestra el detector en forma de tiburón martillo y tres globos livianos unidos entre sí levitando sobre otro globo cargado. Los globos livianos corresponden a globos de agua inflados con aire y cargados por frotación, el globo inferior es un globo común cargado por frotación. Al ubicar los globos pequeños sobre un globo cargado con la misma polaridad, los globos pequeños se alejan entre sí y se mantienen flotando por el aire. Es así posible mover el globo por debajo de los tres globos para mantenerlos volando por el aire frente a todo el curso. Es también posible levitar un trozo de bolsa plástica muy delgada que se expande en 
forma de anillo al ser cargada por frotación. El detector muestra que el globo inferior se encuentra cargado con un exceso de carga negativa.

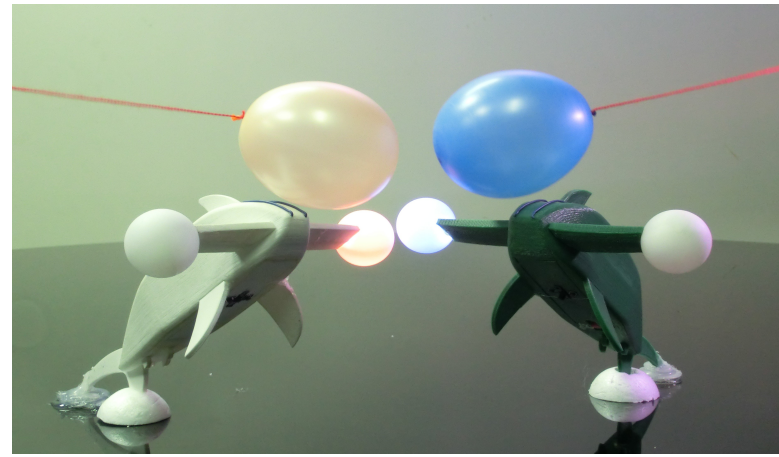

Figura 6. Globos con cargas de distinto signo que se atraen mutuamente.

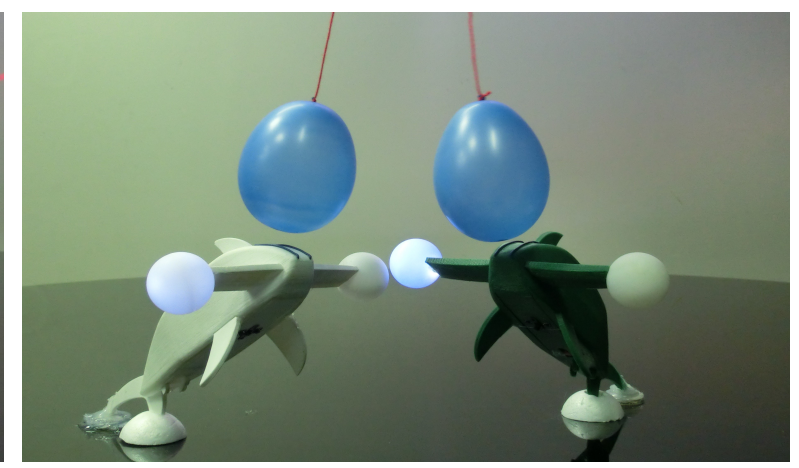

Figura 7. Globos que se repelen debido a un exceso de carga negativa.

Atracción y repulsión de globos cargados por fricción. Sin dudas, una de las actividades más simples de realizar como una primera aproximación para intentar entender la fuerza de interacción eléctrica entre objetos consiste en evidenciar las fuerzas de atracción y repulsión usando globos cargados por fricción. El detector de polaridad puede ser usado para identificar la presencia y signo de las cargas en cada uno de los globos que interactúan y permite evidenciar que la fuerza atractiva se produce entre objetos cargados con distinta polaridad o entre un objeto cargado y uno neutro. El detector permite también evidenciar que la fuerza repulsiva se produce entre objetos cargados con una misma polaridad.

Los experimentos mostrados en las figuras 6 y 7 son muy fáciles de replicar y sólo se debe tener presente que usamos globos pequeños debido a que su bajo peso permite evidenciar claramente la presencia de fuerzas eléctricas. Antes de realizar estos experimentos se deben probar distintos tipos de objetos con los cuales se pueda frotar fácilmente a los globos y obtener distintos signos de cargas en éstos. Es importante que al construir las actividades para su uso en el aula se incluyan etapas en donde los alumnos predigan qué va a suceder y luego de que los alumnos armen experimentos similares a los mostrados, ellos hagan uso del detector de polaridad para identificar el origen de cada fuerza. El detector puede así transformar actividades que usualmente son de tipo demostrativo, en una serie de actividades que permitan indagar el rol del signo de la carga en la fuerza de interacción eléctrica.

Flujos y signos de carga en un electroscopio. El electroscopio es un dispositivo muy simple y es el instrumento en que se basó gran parte del desarrollo inicial de la electrostática. En la versión que usaremos de este dispositivo, se hace evidente la repulsión entre tres esferas conductoras livianas cuando éstas se cargan con una misma polaridad. Estos electroscopios son muy simples de construir usando esferas de poliestireno expandido recubiertas con polvo de grafito y sólo hay que tener cuidado en limpiar los frascos con alcohol y secarlos usando aire caliente para que el exceso de carga se mantenga durante el tiempo necesario para realizar la clase.

La imágenes mostradas en las figura 8 y 9 muestran que las esferas conductoras dentro de cada electroscopio se repelen mutuamente en electroscopios cargados con distinta polaridad y los detectores permiten comprobar los distintos signos para los excesos de carga en cada electroscopio.

El uso de detectores de polaridad en conjunto con el uso de dos electroscopios (Ohriner 1969) permite que los alumnos investiguen una gran diversidad de interacciones y flujos de carga entre objetos cargados. Es posible por ejemplo cargar los dos electroscopios con la misma polaridad o con polaridades distintas y observar que las esferas conductoras dentro de 


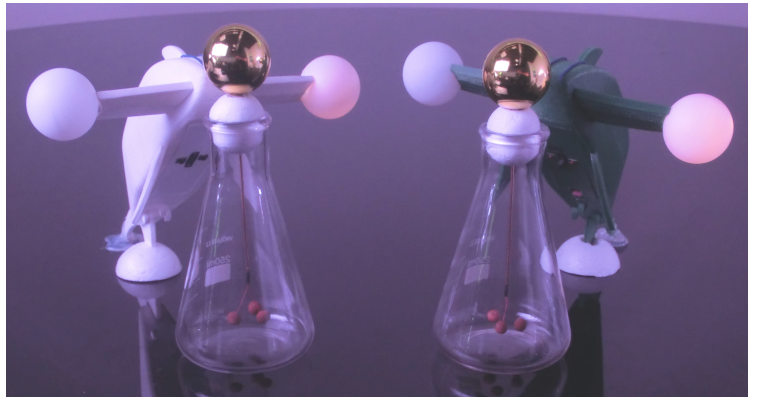

Figura 8. Electroscopios cargados con un exceso de carga positiva.

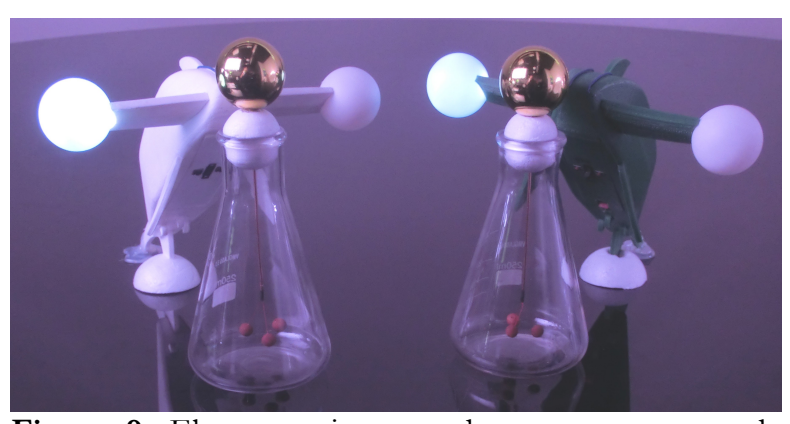

Figura 9. Electroscopios cargados con un exceso de carga negativa.

cada electroscopio se repelen. El detector de polaridad permite visualizar que tanto las esferas cargadas positivas o negativas se repelen entre sí. Esta detección del signo de las cargas podría realizarse luego de permitir a los alumnos hacer un cortocircuito entre los dos electroscopios cargados usando un conductor sujeto por un material aislador. Los alumnos pueden así descubrir que aunque cada electroscopio muestre una repulsión entre sus esferas, al realizar una conexión entre ellos, en un caso se produce un flujo de carga entre electroscopios de distinta polaridad que elimina el efecto de repulsión y en el caso de electroscopios de una misma polaridad no se produce un flujo de carga.

Motor electrostático. Los motores eléctricos modernos son en realidad motores electromagnéticos cuyo principio de funcionamiento se basa en la fuerza de interacción entre campos magnéticos y corrientes eléctricas. Mucho antes de la invención de estos motores ya se habían creado máquinas cuyo movimiento se produce debido a la fuerza de interacción entre objetos cargados (Jefimenko 1973). Describiremos aquí un motor electrostático muy simple de construir que es similar a las electrical wheels inventadas por Benjamin Franklin. Usaremos el detector de polaridad para evidenciar la presencia y signo de las cargas eléctricas en las esferas que componen el motor, y así poder entender el principio de funcionamiento de uno de los primeros motores eléctricos inventados por el hombre.
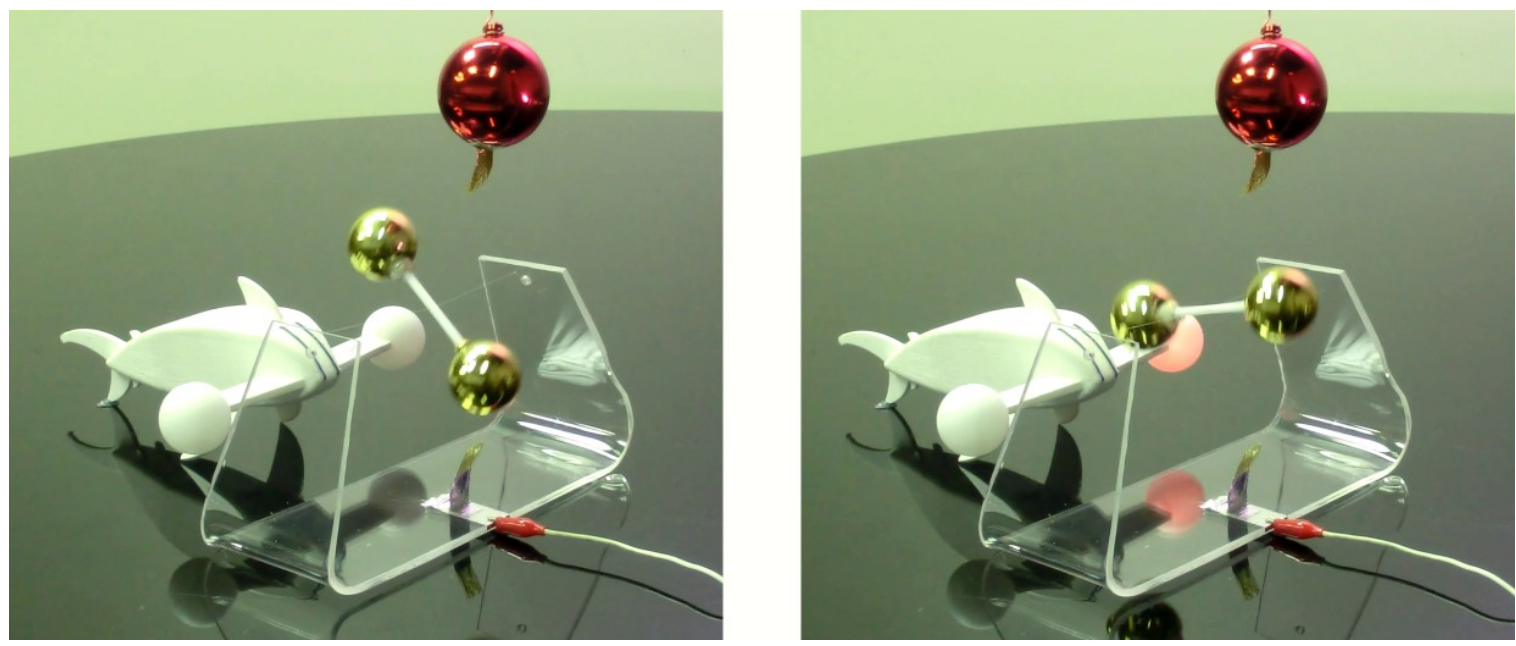

Figura 10. Motor electrostático construido usando dos esferas metalizadas que se cargan de manera alternada al pasar cerca de la esfera superior, la cual obtiene carga desde un generador de Van de Graaff.

La figura 10 muestra un motor electrostático muy simple de construir usando dos esferas de plástico metalizadas como las usadas para adornar árboles en navidad. Para construir un motor de este tipo se necesitan esferas conductoras que transfieran la carga desde el generador de Van de Graaff hacia el conector conectado a tierra. En todas las actividades mencionadas en 
este trabajo los objetos cargados adquieren potenciales eléctricos de varios miles de volts pero en donde la cantidad de carga acumulada no es suficiente para producir daños a las personas. Sin embargo, los grandes voltajes involucrados permiten producir chispas y traspasos de carga incluso en objetos considerados normalmente aisladores de la electricidad. En el caso de las esferas ornamentales de navidad, las esferas están construidas de plástico no conductor con una capa metálica conductora en su exterior, que está recubierta de una delgada capa no conductora. Los grandes voltajes producidos por el Van de Graaff permiten que la carga fluya fácilmente a través de la delgada capa aisladora que recubre a las esferas.

El funcionamiento de estos primeros motores eléctricos se basa en la atracción que se produce entre un objeto fijo cargado con alto voltaje (en nuestro caso la esfera superior conectada a un Van de Graaff) que atrae a un objeto conductor descargado y libre de moverse, esta atracción produce movimiento del objeto móvil, el que luego de tocar al objeto fijo (o pasar muy cerca de él) se carga con la misma polaridad de éste y es repelido hacia un contacto a tierra. Luego de que el conductor móvil pasa muy cerca o toca el contacto a tierra, éste se descarga y el ciclo comienza nuevamente. Típicamente este tipo de motor usa dos o más esferas conductoras móviles. Los motores electrostáticos no han sido adoptados en nuestra sociedad debido a que requieren que los objetos se carguen a altos voltajes y pueden producir chispas de descarga hacia cualquier objeto cercano.

\section{Conclusiones}

Nuestro universo es gobernado por cuatro fuerzas fundamentales y debido a que dos de ellas se manifiestan en la escala nuclear, nuestros sentidos sólo pueden acceder directamente a manifestaciones de las fuerzas de gravedad y eléctrica. La fuerza de gravedad es extremadamente débil y sólo podemos sentir sus efectos debido a la gran masa de nuestro planeta. Debido a que la fuerza de interacción gravitacional entre dos objetos de un tamaño del orden de la escala humana es prácticamente nula, la mayoría de los efectos que explican nuestro mundo cotidiano son de origen eléctrico. Es así de suma importancia contar con experimentos que permitan a los alumnos investigar de manera simple las características de las cargas eléctricas y sus interacciones.

Un detector de polaridad construido a partir de componentes electrónicos no es algo nuevo (Dinca 2011, Isik 2015, Ganci 1994, Thompson 2014, Connolly 1991) y es también posible medir el signo de la carga usando una ampolleta de Neón (Chen 1999) o un electrómetro comercial. Lamentablemente, las ampolletas de Neón modernas no permiten visualizar claramente cual electrodo es el que se ilumina con la presencia de altos voltajes y los electrómetros no son muy comunes en un laboratorio de ciencias. Para el diseño de nuestro detector nos hemos preocupado de que el circuito mostrado en la figura 2 sea simple y su montaje mostrado en la figura 4 sea fácil de entender y de armar. Este detector ha sido usado en conjunto con las actividades mencionadas en el texto como experimentos demostrativos al inicio de un laboratorio de electrostática para alumnos de segundo año universitario de la carrera de física. Aunque el uso de este detector no ha sido escalado para que los alumnos lo usen en sus actividades de laboratorio, es evidente que el detector pasará a ser un elemento imprescindible en futuras versiones del curso. Este uso incipiente del detector ha provocado que los profesores de física de enseñanza media que han visto el detector funcionando, con su cuerpo impreso en forma de tiburón, han comenzado a pedir que les imprimamos uno para llevárselo a sus escuelas. Esperamos que en un futuro cercano nuestro diseño novedoso de un detector en forma de tiburón martillo permita capturar la atención de alumnos de todas las edades, para que actividades experimentales como las propuestas en este trabajo los guíen 
hacia una comprensión funcional del origen de las interacciones entre objetos cargados eléctricamente.

\section{Agradecimientos}

Este trabajo fue financiado por el Fondo Nacional de Desarrollo Científico y Tecnológico FONDECYT (proyecto $\mathrm{N}^{\circ}$ 1151257) y por la Dirección General de Investigación y Posgrado de la Pontificia Universidad Católica de Valparaíso.

\section{Referencias}

Ampullae (2017) Ampullae of Lorenzini. Wikipedia.

Beaty W. J. (1987) Ridiculously Sensitive Electric Charge Detector.

Chen X. Y., Wang X. (1999) Detecting the Polarity of an Electric Charge. The Physics Teacher 37 (6), 344.

Connolly W., Benoit G., Bould M. (1991) Apparatus for teaching physics: A new device for studying electric fields. The Physics Teacher 29 (3), 182-185.

Dinca M. P. (2011) Charge sniffer for electrostatic demonstrations. American Journal of Physics 79 (2), 217-221.

FreeCAD (2017) FreeCAD.

Kriman B., Nasyrov R. (2008) Electrostatic toy.

Ganci S. (1994) An electroscope discriminating the sign of charges. American Journal of Physics $62(5), 474$.

Isik H. (2015) An instrumental method to identify electric charge types with a simple device. Physics Education 50 (4), 393-396.

Jefimenko O. D. (1973) Electrostatic Motors. Their History, Types, and Principles of Operation. Star City. Electret Scientific Company.

Laws P. W. (2009) Physics with Video Analysis. Vernier Software and Technology.

Lozano O. R. (2013) La ciencia recreativa como herramienta para motivar y mejorar la adquisición de competencias argumentativas. Enseñanza de las Ciencias 31 (3), 265-273.

Ohriner M., Machtinger S. (1969) Two electroscopes are better than one. The Pbysics Teacher 7 (6), 343-344.

Thompson F. (2014) An electronic electroscope. Physics Education 49 (1), 8-20.

Vera F., Romanque C. (2009) Another way of tracking moving objects using short video clips. The Physics Teacher 47, 370-373.

Vera F., Rivera R., Ortiz M. (2014) A simple experiment to measure the inverse square law of light in daylight conditions. European Journal of Physics 35, 015015. 